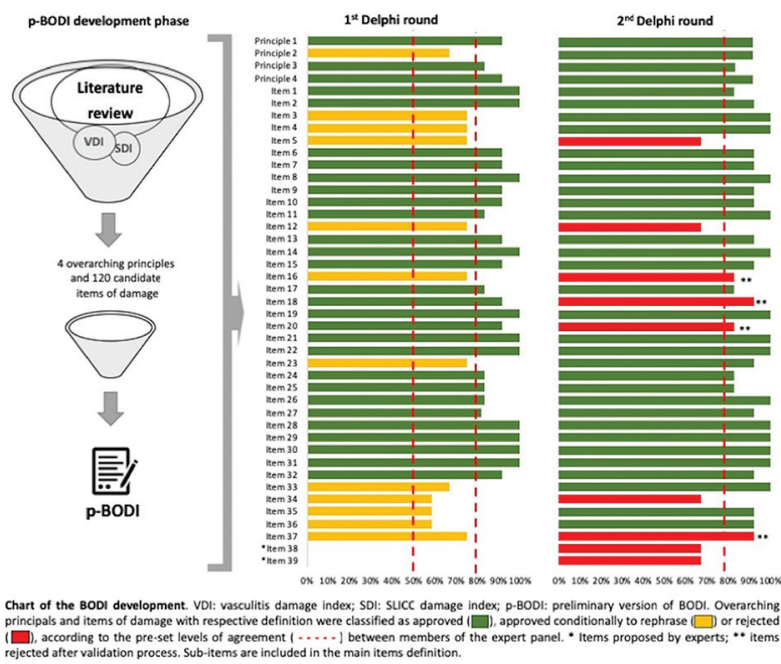

Disclosure of Interests: Matteo Piga: None declared, Alberto Floris: None declared, Gerard Espinosa: None declared, Nikolaos Kougkas: None declared, Andrea Lo Monaco: None declared, Giuseppe Lopalco Speakers bureau: SOBI, BMS, Ida Orlando: None declared, Vittorio Pirani: None declared, Ernestina Santos: None declared, Luísa Serpa Pinto: None declared, George Bertsias: None declared, Luca Cantarini: None declared, Alberto Cauli: None declared, Ricard Cervera: None declared, João Correia: None declared, Marcello Govoni: None declared, Florenzo lannone Consultant for: $\mathrm{F}$ lannone has received consultancy fees and/or speaker honoraria from Pfizer, AbbVie, MSD, BMS, Novartis, Lilly, UCB outside this work, Speakers bureau: F lannone has received consultancy fees and/or speaker honoraria from Pfizer, AbbVie, MSD, BMS, Novartis, Lilly, UCB outside this work, Ana Martins da Silva: None declared, Piergiorgio Neri: None declared, Carlos Vasconcelos: None declared, Monica Muntoni: None declared, Alessandro Mathieu: None declared

DOI: 10.1136/annrheumdis-2019-eular.3517

\section{SAT0241 HEALED TEMPORAL ARTERITIS. ANALYSIS OF PATIENTS REFERRED TO RHEUMATOLOGY WITH A DIAGNOSIS OF TEMPORAL ARTERITIS}

Lily Romero Karam ${ }^{1}$, Shruti Agashe ${ }^{2}$, lleana De Anda-Duran ${ }^{3}$, Ramona Mihu ${ }^{4}$, Jovan Popovich ${ }^{4} .{ }^{1}$ Houston Methodist, Internal Medicine, Houston, United States of America; ${ }^{2}$ Houston Methodist, Neurology, Houston, United States of America; ${ }^{3}$ Harvard T.H. Chan School of Public Health, MPH-Epidemiology, Boston, United States of America; ${ }^{4}$ Houston Methodist, Rheumatology, Houston, United States of America

Background: The diagnosis of giant cell arteritis (GCA), or temporal arteritis (TA), is a clinical one. Temporal artery biopsy is usually obtained to strengthen support for treatment. Unfortunately a biopsy does not always confirm the diagnosis; results can be reported as active, healed, or negative even in patients with all other features of giant cell arteritis. Histopathologic features suggestive of resolving inflammation are evident in healed TA, but these are non-specific and can also be seen in other conditions that are unlikely to benefit from prolonged steroid treatment. It is not clear if a pathological diagnosis of healed TA helps to support treatment which may be associated with significant toxicity.

Objectives: We analyzed clinical features of patients with "healed" TA and compared them to patients with active and negative biopsies with the goal of identifying any features that may aid treatment decisions.

Methods: Retrospective analysis of 49 patients with established TA diagnosis, seen at a rheumatology practice in Houston, Texas from 8/2007 to 12/2018. Fifty seven percent were referred by Ophthalmology, the rest by other specialties. Patients were divided into 3 groups based on biopsy: Active, Healed, and Negative. Clinical features analyzed at the time of presentation were gender, age of onset, fulfillment of the American College of Rheumatology (ACR) 1990 criteria for the classification of Giant Cell Arteritis and the 2016 revised ACR criteria for early diagnosis of Giant Cell Arteritis (rACR), and the presence of vision abnormalities, headache, temporal artery tenderness/induration, jaw claudication, fever, anemia, sedimentation rate at onset, and flares during follow up.

Descriptive data was reported as means \pm standard deviation for continuous variables and as proportions for categorical variables; analyzed with ANOVA and chi squared, respectively. Post hoc analysis with Bonferroni corrections and Fisher's exact tests were used. All analyses were performed on Stata version 15 (StataCorp LP, College Station, TX, USA). A p-value of $\leq 0.05$ was considered statistically significant.

Results: Thirty-two (65\%) of the 49 patients were female and 17 (35\%) were male. Biopsy reports showed active disease in 14 (28.5\%), healed in $31(63.3 \%)$ and negative in $4(8.2 \%)$ patients. Average age was 72.7 years $\pm 6.3,73.0 \pm 9.1,61.25 \pm 1.3$ years in these 3 groups, respectively ( $p$ value 0.008 ). The Active versus Healed groups were statistically different with respect to age ( $p$ 0.028), those meeting ACR and rACR criteria ( $p$ 0.029 and $p$ 0.002), and the incidence of jaw claudication: 8 active $(57.1 \%)$ versus 5 healed $(16.1 \%)$ patients ( $p$ 0.05). There was no statistically significant difference in ophthalmologic manifestations, headache, temporal artery palpation, fever, anemia, and flares. In addition, $90 \%$ of patients reported side effects from steroids.

Conclusion: The percent of healed TA was $63.3 \%$, higher than in other studies. Patients with healed TA were less likely to fulfill ACR and rACR criteria for giant cell arteritis. Further studies are needed to identify the subset of patients with healed TA who would have a clear benefit from high dose steroids, that would outweigh the treatment side effects. At the end of the day, the diagnosis of TA remains a clinical one and early involvement of Rheumatology may help avoid unnecessary biopsies and treatment. Ultrasound, MRA, and PET-CT scan may be able to fill the gap when biopsy fails to confirm the diagnosis.

Disclosure of Interests: None declared

DOI: 10.1136/annrheumdis-2019-eular.3559

\section{SAT0242 SENSITIVITY OF TEMPORAL ARTERY BIOPSY IN GIANT CELL ARTERITIS: SYSTEMATIC LITERATURE REVIEW AND META-ANALYSIS OF CLINICAL DATA}

Emma Rubenstein ${ }^{1}$, Carla Maldini ${ }^{1}$, Solange Gonzalez-Chiappe ${ }^{1}$, Sylvie Chevret $^{2}$, Alfred Mahr'. ' Saint Louis University Hospital, Department of Internal Medicine, Paris, France; ${ }^{2}$ Saint Louis University Hospital, Department of Biostatistics, Paris, France

Background: The role of temporal artery biopsy (TAB) as a reference test for the diagnosis of giant cell arteritis (GCA) is currently questioned by the use of non-invasive imaging techniques such as temporal artery ultrasonography (TA-US). Although TAB is highly specific, a subset of patients with a clinical diagnosis of GCA does not show the characteristic histopathological signs. The lack of knowledge of the proportion of GCA cases with positive findings on TAB hampers comparisons of the sensitivity of TAB and imaging tests for diagnosing GCA.

Objectives: We performed a systematic literature review and meta-analysis to estimate the sensitivity of TAB in GCA and to identify factors that may influence the estimate.

Methods: We searched MEDLINE via PubMed, EMBASE and CENTRAL databases for articles reporting $T A B$ in $G C A$ that were published from 1990 to 2017, with no language restriction. Eligibility criteria included studies with $\geq 30$ GCA cases fulfilling the original or modified 1990 ACR classification criteria for GCA. From eligible publications, two independent researchers extracted the main methodological, geographic, demographic, and clinical characteristics and the number of TAB-positive cases among all cases with interpretable results for TAB. By meta-analysis, we computed the pooled proportion of TAB-positive GCA cases by using a random-effects model with a binomial-normal distribution and assessed heterogeneity by the $\mathrm{I}^{2}$ statistic. Subgroup and meta-regression analyses were used to examine the effect of 16 covariates (e.g., geographic, demographic, clinical and study descriptors) on TAB positivity.

Results: Among 3820 screened publications, 32 independent studies (3092 GCA patients in total) were used for the analysis. The pooled proportion of TAB positivity was estimated at $77.3 \%(95 \%$ confidence interval $71.8-81.9 \%)$, with high between-study heterogeneity $\left(F^{2}=90 \%\right)$. Subgroup analysis suggested a potential influence of year of publication (Table). This result was confirmed by univariate $(P=0.0008)$ and multivariate metaregression $(P=0.0004)$. No other analyzed covariate significantly influenced the sensitivity of TAB in GCA.

Conclusion: The $77 \%$ estimated sensitivity of TAB in GCA indicates that it is not inferior to that of TA-US (1). The decline in TAB-positive GCA cases over time could reflect an increasing propensity of clinicians to accept GCA diagnosis in the absence of proof by TAB. The unexplained high between-study heterogeneity could also result from differences in $\mathrm{TAB}$ sampling, processing or interpretation.

\section{REFERENCE}

[1] Duftner C, Dejaco C, Sepriano A, et al. Imaging in diagnosis, outcome prediction and monitoring of large vessel vasculitis: a systematic literature review and meta-analysis informing the EULAR recommendations. RMD Open. 2018;4:e000612. 
Table. Proportion of TAB-positive GCA stratified by the main covariates.

\begin{tabular}{lcccc}
\hline Study and pt. characteristics & & $\begin{array}{c}\text { No. of studies } \\
\text { (pt.) }\end{array}$ & $\begin{array}{c}\text { Positive TAB } \\
(\%) \\
(95 \% \mathrm{Cl})\end{array}$ & $\mathrm{I}^{2}(\%)$ \\
\hline Mean age at diagnosis & $<72.7$ yrs & $10(961)$ & $81.2(71.1-88.4)$ & 91 \\
\hline$\geq 72.7$ yrs & $11(1067)$ & $74.8(65.1-82.6)$ & 91 & \\
Females & $<71 \%$ of pt. & $12(1156)$ & $78.3(67.0-86.5)$ & 94 \\
& $\geq 71 \%$ of pt. & $11(1319)$ & $81.6(73.9-87.4)$ & 88 \\
Polymyalgia rheumatica & $<42 \%$ of pt. & $7(629)$ & $70.6(59.4-79.9)$ & 87 \\
& $\geq 42 \%$ of pt. & $7(1024)$ & $79.2(66.2-88.0)$ & 94 \\
Ophthalmological signs & $<21 \%$ of pt. & $6(893)$ & $81.8(66.2-91.2)$ & 95 \\
& $\geq 21 \%$ of pt. & $7(606)$ & $72.2(60.4-81.6)$ & 87 \\
Large-vessel & $<25 \%$ of pt. & $2(373)$ & $63.0(50.6-73.8)$ & 78 \\
involvement & $\geq 25 \%$ of pt. & $2(203)$ & $61.1(54.2-67.5)$ & 0 \\
Study purpose & Diagnostic & $3(153)$ & $88.8(68.5-96.7)$ & 75 \\
Study type & Other & $29(2939)$ & $76.0(70.4-80.9)$ & 90 \\
& Retrospective & $24(2688)$ & $75.5(69.3-80.8)$ & 91 \\
Year of publication & Prospective & $8(404)$ & $83.1(70.6-90.9)$ & 86 \\
& $<2012$ & $16(1806)$ & $84.4(77.1-89.6)$ & 92 \\
& $\geq 2012$ & $16(1286)$ & $68.4(63.4-72.9)$ & 69 \\
\hline
\end{tabular}

Pt : patients

Disclosure of Interests: Emma Rubenstein: None declared, Carla Maldini: None declared, Solange GONZALEZ-CHIAPPE: None declared, Sylvie Chevret: None declared, Alfred Mahr Consultant for: Chugai Pharma France, Speakers bureau: Roche SAS Chugai Pharma France DOI: 10.1136/annrheumdis-2019-eular.4204

\section{SAT0243 EFFECTS OF TOFACITINIB SUPPRESSED PULMONARY VASCULAR REMODELING OF ALLERGIC VASCULITIS IN A MURINE MODEL}

Nobuhito Sasaki, Kohei Yamauchi. Iwate Medical University School of Medicine, Morioka, Japan, Division of Pulmonary medicine, Allergy and Rheumatology, Department of Internal Medicine, Morioka, Japan

Background: We reported allergic granulomatous vasculitis with eosinophil infiltration in an asthma model of $\mathrm{C} 57 \mathrm{BL} / 6$ sensitized with ovalbumin (OVA). TGF-btea and IL-6 are thought to play an important role in fibroblasts proliferation and is critical to vascular remodeling in vasculitis. Tofacitinib inhibits vascular endothelial cells proliferation and canalization. Objectives: To elucidate the role of tofacitinib in vascular remodeling of allergic granulomatous vasculitis, we examined the effects of tofacitinib on the vasculitis of the murine model.

Methods: C57BL/6 mice (6-8 weeks) were sensitized with ovalbumin (OVA) and alum. The positive controls $(n=9)$ were exposed to aerosolized OVA daily for 7 days. The other group of mice (tofacitinib treated mice $(n=9))$ were administered with tofacitinib $(100 \mathrm{mg} / \mathrm{kg}$ intraperitoneal administration) in parallel with daily exposure to aerosolized OVA for 7 days. On 7th day, bronchoalveolar lavage (BALF) was performed and the lungs were excised for pathological analysis. Cytokines in BALF were measured.

Results: The total cell number and the number of. Eosinophils in BALF on the 7th day were decreased significantly in the tofacitinib-treated mice compared with those of the control-positive mice. The blood eosinophil counts in the positive control increased after OVA inhalation. The blood eosinophil counts in the tofacitinib treated mice were lower on the than those in the positive control.
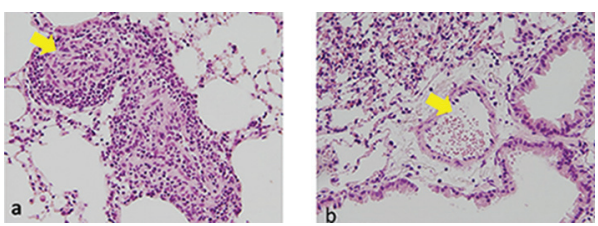

Figure 1. a. Positive control: Totally occluded pulmonary artery by intraluminal myofibroblasts in the OVA-sensitized mice with exposure to OVA in 7th day. (HE staining) b. Tofacitinib: Intraluminal myofibroblast accumulation was not observed in the OVA-sensitized mice with exposure to OVA and treated with tofacitinib in 7th day. (HE staining).

The concentrations of IL-4, IL-5, IL-6 and TGF-beta in BAL fluids reduced significantly in the tofacitinib treated group. The pathological scores reduced significantly in the tofacitinib treated group compared to the positive control group. Intra luminal infiltration and proliferation of Ki67 positive myofibroblasts, IL- 6 positive cell and $\alpha$-SMA positive cells in pulmonary arteries were reduced dramatically in the tofacitinib treated group compared to the positive control group.

Conclusion: Tofacitinib suppressed pulmonary vascular remodeling in a murine model of allergic vasculitis with eosinophil infiltration. Tofacitinib is a hopeful therapeutic drug for Eosinophilic granulomatosis with polyangiitis.

Disclosure of Interests: None declared

DOI: 10.1136/annrheumdis-2019-eular.4151

\section{SAT0244 EXPANDING SPECTRUM OF ADENOSINE DEAMINASE 2 (DADA2) MANIFESTATIONS: EXPERIENCE 13 PATIENTS FROM INDIA}

Aman Sharma ${ }^{1}$, Gsrsnk Naidu ${ }^{1}$, Ramesh Jois ${ }^{2,3}$, Chengappa Kavadichanda ${ }^{4}$, Rajkiran Dudam ${ }^{5}$, Sanjay Jain ${ }^{1}$, Vir Negi ${ }^{4}$, Pui.Y Lee ${ }^{6}{ }^{1}$ Post Graduate Institute of Medical Education and Research, Chandigarh, Internal Medicine, Chandigarh, India; ${ }^{2}$ Vikram Hospital, Bengaluru, India; ${ }^{2}$ Vikram Hospital, Bengaluru, India; ${ }^{4}$ Jawaharlal Institute of Postgraduate Medical Education and Research, Puducherry, India; ${ }^{5}$ Hyderabad Rheumatology Center, Hyderabad, India; ${ }^{6}$ Boston Children's hospital, Boston, United States of America

Background: Deficiency of adenosine deaminase 2 (DADA2) is a recently described hereditary autoinflammatory disease with limited published literature [1-5]. There are no previous case series published from Asia.

Objectives: To describe the clinical features and treatment responses of patients diagnosed with DADA2 from India.

Methods: Patients diagnosed with DADA2 at 4 different centres in India were included. Details of clinical features, laboratory investigations, ADA2 activity, genetic analysis of CECR 1 gene, therapy received and outcomes were noted.

Results: A total of 13 patients (12 Indians and one Syrian) were diag nosed with DADA2 between April 2017 and January 2019. The median age was 22 years (range: $7-39$ years) and 8 (61.5\%) patients were males. The diagnosis was made at a median duration of 30 months (range: 1-360 months) from the onset of first symptom. Figure 1 shows the various clinical manifestations. One patient had unknown manifestation of bad obstetric history mimicking APLA syndrome. ESR (median: 49.5 $\mathrm{mm} / \mathrm{hr}$; range: $15-130 \mathrm{~mm} / \mathrm{hr}$ ) was raised in all except one patient who was in remission at the time of diagnosis. CRP value was available in 11 patients and was elevated in all (median: $51.4 \mathrm{mg} / \mathrm{L}$; range: 17.6-140 $\mathrm{mg} / \mathrm{L})$. Immunoglobulin levels assessed in 4 patients were normal. Neuroimaging done in nine patients showed infarcts in 5 , bleed in 3 , multiple aneurysms and features of PRES in one patient each. Abdominal microaneurysms were noted in 5 patients. Arterial occlusion of peripheral limb vessels was noted in 2 patients. ADA2 activity was assessed in 9 patients and all had nearly undetectable activity. CECR1 gene mutation analysis was available in 9 patients: 8 patients were homozygous for $p$ (Gly47Arg) missense mutation at exon 2 of CECR1 gene while one patient displayed compound heterozygosity for two novel mutations, $P$ (Leu188Val) in exon 4 and C.753+2 $\mathrm{T}>\mathrm{A}$ in intron 4 of CECR1 gene. Eight patients were started on anti-TNF drugs (adalimumab in 7 and etanercept in 1). One patient required higher doses of adalimumab (40mg followed by $120 \mathrm{mg}$ at week 1 and $80 \mathrm{mg}$ at week 2) for severe gastrointestinal involvement but later died due to disseminated herpes infection. Rest of the seven patients started on anti-TNF drugs achieved disease control. Our patients had CNS, eye and Gl manifestations lower than those reported by Zhou et $\mathrm{al}^{1}$ but higher than those reported by Nanthapisal et $\mathrm{al}^{2}$ and Navon Elkan et $\mathrm{al}^{4}$

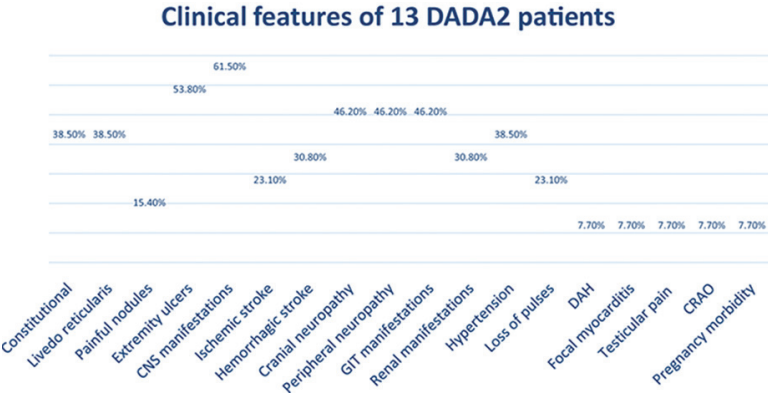

Figure 1. Clinical manifestations noted in our cohort of 13 patients with DADA2. 